Zygote 23 (August), pp. 556-562. (c) Cambridge University Press 2014. The online version of this article is published within an Open Access environment subject to the conditions of the Creative Commons Attribution-NonCommercial-ShareAlike licence <http:/ / creativecommons.org/licenses/by-nc-sa/3.0/ > . The written permission of Cambridge University Press must be obtained for commercial re-use.

doi:10.1017/S0967199414000288 First Published Online 3 July 2014

\title{
A double-blinded comparison of in situ TUNEL and aniline blue versus flow cytometry acridine orange for the determination of sperm DNA fragmentation and nucleus decondensation state index
}

\author{
Jamal Hamidi ${ }^{2}$, Christophe Frainais ${ }^{2}$, Edouard Amar ${ }^{3}$,Eric Bailly ${ }^{4}$, Patrice Clément ${ }^{2}$ \\ and Yves Ménézo $0^{1,3,4,5}$ \\ Laboratoire Clément, Paris France; Urology Department, American Hospital of Paris, Neuilly-sur-Seine, France; Beckman \\ Coulter France, Villepinte, Roissy, France; and Biological Reproductive Products (BRP), Lugano, Switzerland
}

Date submitted: 22.12.2013. Date revised: 31.3.2014. Date accepted: 1.4.2014

\section{Summary}

The impact of sperm DNA fragmentation on assisted reproductive technology (ART) successes, in terms of outcome, is now established. High levels of DNA strand breaks severely affect the probability of pregnancy. The importance of sperm nucleus condensation in early embryogenesis and, subsequently, on the quality of the conceptus is now emerging. In this article we have compared in situ analyses with terminal deoxynucleotidyl transferase (TdT)-mediated dUTP nick end labelling (TUNEL) (for DNA fragmentation) with aniline blue (AB) (for nucleus decondensation), versus flow cytometry (FC) after acridine orange staining, in a double-blinded analysis. In our hands, TUNEL and acridine orange give perfectly comparable results. For decondensation the results are also comparable, but the double-stranded green fluorescence obtained with acridine orange seems to slightly underestimate the decondensation status obtained with $\mathrm{AB}$.

Keywords: Acridine orange, Aniline blue, Decondensation, DNA fragmentation, Flow cytometry, Sperm, TUNEL

\section{Introduction}

According to the World Health Organization (WHO) (2004) estimation, infertility may now affect one couple of five in western countries. Male reproductive failure is thought to be the cause of at least onehalf of the total number of failures. During the past half century and before the advent of large scale in vitro fertilization, routine semen analysis was oriented towards numeration, concentration, motility

\footnotetext{
${ }^{1}$ All correspondence to: Yves Ménézo. Laboratoire Clément, 17 avenue d'Eylau, 75016 Paris and 8 avenue Henri Barbusse, 93150 Le Blanc Mesnil, France. Tel: +33 1 48670257. Fax: +33 1 48670360. e-mail: yves.menezo@gmail.com

${ }^{2}$ Laboratoire Clément, 17 avenue d'Eylau, 75016 Paris and 8 avenue Henri Barbusse, 93150 Le Blanc Mesnil, France.

${ }^{3}$ Urology Department, American Hopsital of Paris, 92200

Neuilly-sur-Seine, France.

${ }^{4}$ Beckman Coulter France, Villepinte, 95942 Roissy, France.

${ }^{5}$ Biological Reproductive Products (BRP), Lugano, Switzerland.
}

and morphology. Rapidly it has become obvious that a better appreciation of sperm chromatin is needed. A 'transitory' interest in nuclear shape and chromatin texture, i.e. the organization of chromatin condensation, measured by aniline blue $(\mathrm{AB})$ staining (Auger et al., 1990) or by chromomycin A3 (Sakkas et al., 1996) has emerged. But overall, interest has been focused on sperm DNA fragmentation (Evenson et al., 1980), especially in relation to oxidative stress. The large waste observed for embryos conceived by assisted reproductive technology (ART) has led to the common consensus that fertilization and embryo development constitute only a small proportion of sperm development capacity, as it is also true that the quality of the first embryo cleavage is under paternal effect (Eid et al., 1994, Ward et al., 2001). True sperm capacity is defined as the ability to sustain embryo development to term. Progress has been made in the global determination of DNA strand fractures (i.e. fragmentation; Evenson et al., 1980) and the identification of specific insults such 
as oxidation products and adducts (Gaspari et al., 2003, Badouard et al., 2008, Ji et al., 2013), essentially those originating from pollution. Interest towards the tertiary structure of the nucleus, i.e. its condensation, is re-emerging (Kim et al., 2013, Sellami et al., 2013). The importance of the structural interactions between DNA and the packaging proteins is no longer a matter for debate: failures in condensation induce delays in the first cell cycle with further detrimental consequences at some point for the developing embryo (Rousseaux et al., 2008; Ward, 2010). This includes recurrent abortion (Kazerooni et al., 2009). This highly compacted structure is not inert. Some gene families that are highly important for early embryo development are associated with histones (in human spermatozoa: Hammoud et al., 2009). If the oocyte has an important, yet finite, DNA repair capacity, it is largely efficient in relation to DNA strand breaks (Menezo et al., 2007, 2010). The oocyte capacity to improve tertiary structure is rather limited. Chromatin assembly factor 1 (CAF-1), and H2AFX (H2A histone family, member $X$ ) are correctly expressed in the oocyte; however it is not clear if these factors are active at the correct time, immediately at fertilization. This means that determination and correction (when possible; Menezo et al., 2014) of decondensation is of paramount importance in ART. There is no real consensus on the technique of evaluation of DNA fragmentation, but most of all for decondensation, which is usually totally neglected. In a first approach, we have demonstrated that chromomycin A and terminal deoxynucleotidyl transferase (TdT)-mediated dUTP nick end labelling (TUNEL) have overlapping characteristics: chromomycin A3 (CMA3) is a DNA dye, a guanine-cytosine-specific fluorochrome that competes with protamines for access to DNA. Aniline blue $(\mathrm{AB})$ is a better marker of the sperm nucleus chromatin decondensation state (Belloc et al., 2009). In this study we have compared, on the same samples, the DNA fragmentation index (DFI) measured by TUNEL in situ and the DNA decondensation state index (SDI) measured by $\mathrm{AB}$ on slides versus flow cytometry (FC) after staining with acridine orange.

\section{Material and methods}

The analyses were performed in a double-blinded manner in 88 patients, who were under consultation for infertility after at least one ART failure. The sperm was collected from patients by masturbation. After liquefaction $(0.5 \mathrm{~h})$, the sperm was aliquoted in $2 \times$ $0.5 \mathrm{ml}$ samples. Samples were frozen in liquid nitrogen until analysis for dosage using TUNEL on slides and FC analyses. For AB testing, the samples were directly analysed after liquefaction according to the technique of Hamadeh et al. (1996) (see also Belloc et al. 2008). In total, 200 sperm cells were examined for each in situ determination.

\section{DNA fragmentation with TUNEL}

For DNA fragmentation with TUNEL, the classical terminal deoxy ribonucleotidyl transferase-mediated uridine $5^{\prime}$-triphosphate-biotin nick-end labelling test was performed. The fluorescein isothiocyanate-labelled deoxyuridine triphosphate (dUTP) kit (Roche Diagnostics Corporation, Mannheim, Germany) was used according to the instructions of the manufacturer for the in situ technique (see Cohen-Bacrie et al., 2009).

\section{Acridine orange staining and flow cytometry}

For acridine orange, flow cytometry (FC), if the sperm concentration was lower than $0.5 \times 10^{6} / \mathrm{ml}$, before acridine testing, the sample was washed/concentrated using a phosphate-buffered medium containing albumin at $10 \mathrm{~g} / \mathrm{l}$, in order to reach a final concentration of at least $1 \times 10^{6} / \mathrm{ml}$ (Menezo, 2003; unpublished data). After thawing, the sperm were diluted in Earles medium $+1 \%$ albumin. For either concentration or dilution, the presence of proteins was necessary in order to avoid agglutination. After dilution $(1 / 10)$ the sperm sample was lysed in a $0.1 \%$ Triton solution in $1 \mathrm{M} \mathrm{HCl}$. After mixing, the staining solution (acridine orange in a phosphate citrate buffer) is added. The suspension was shaken gently for $30 \mathrm{~s}$ before introduction into the cytometer. The number of cells analysed was roughly 8000 . Sperm samples were thawed and prepared one by one at the last minute. This step is necessary to maintain reproducibility.

The samples were analysed on a flow cytometer FC500 Beckman Coulter equipped with a BioSense $\mathrm{TM}^{\mathrm{TM}}$ measurement cell $(150 \mu \mathrm{M} \times 450 \mu \mathrm{M})$. The laser used was an Argon device $488 \mathrm{nM}$, with seven detection units, that was focused on a elliptic measurement area $10 \mu \mathrm{M} \times 80 \mu \mathrm{M}$. The flow reproducibility and reliability was controlled for each measurement using flow check microspheres. The optical fluorescence stability detection was controlled using Flowser microspheres. Quantification was realized by gathering the signals of sperm cells obtained at $525 \mathrm{~nm}$ for SDI and at $620 \mathrm{~nm}$ for the lesions of single DNA strands (DFI).

\section{Statistical analysis}

One of the comparison of the data obtained was performed using the Welch two-sample $t$-test; or an alternative two-sided test. The other was performed using the Wilcoxon test. 


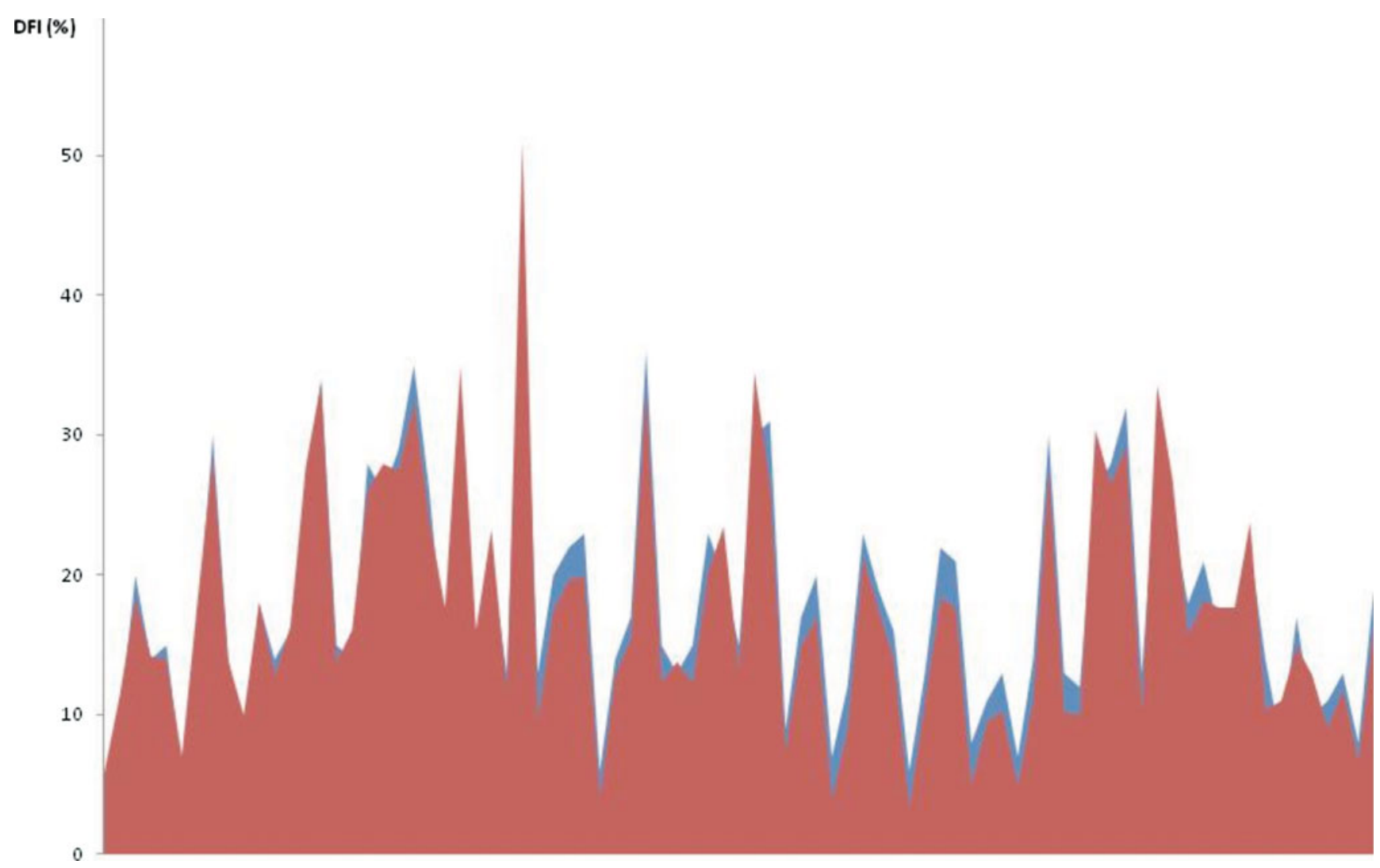

Figure 1 In situ TUNEL versus acridine orange flow cytometry. Individual variations: blue, TUNEL; red, acridine orange.

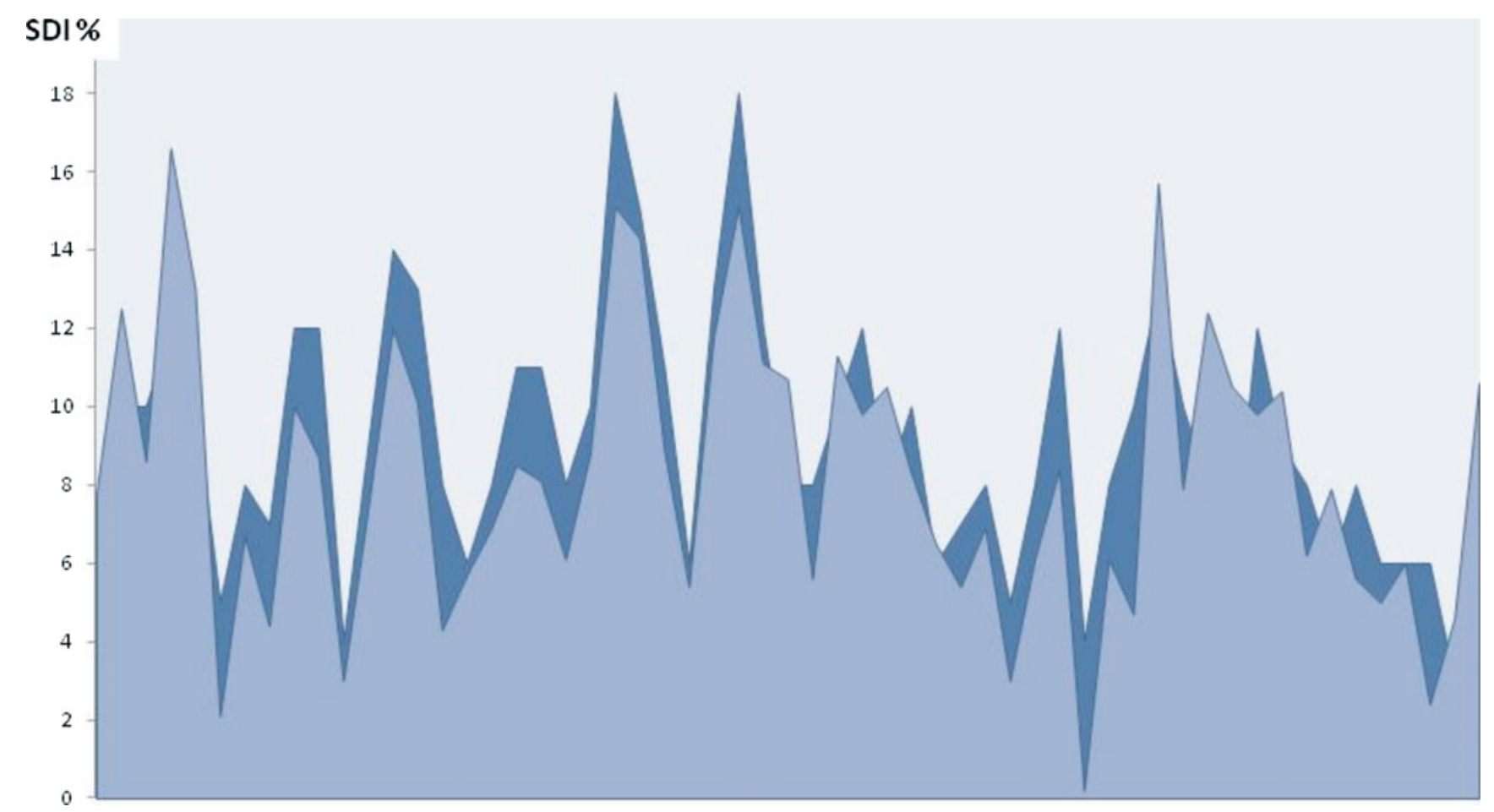

Figure 2 In situ aniline blue versus acridine orange flow cytometry. Individual variations: dark blue: aniline blue; light blue: acridine orange green fluorescence. 


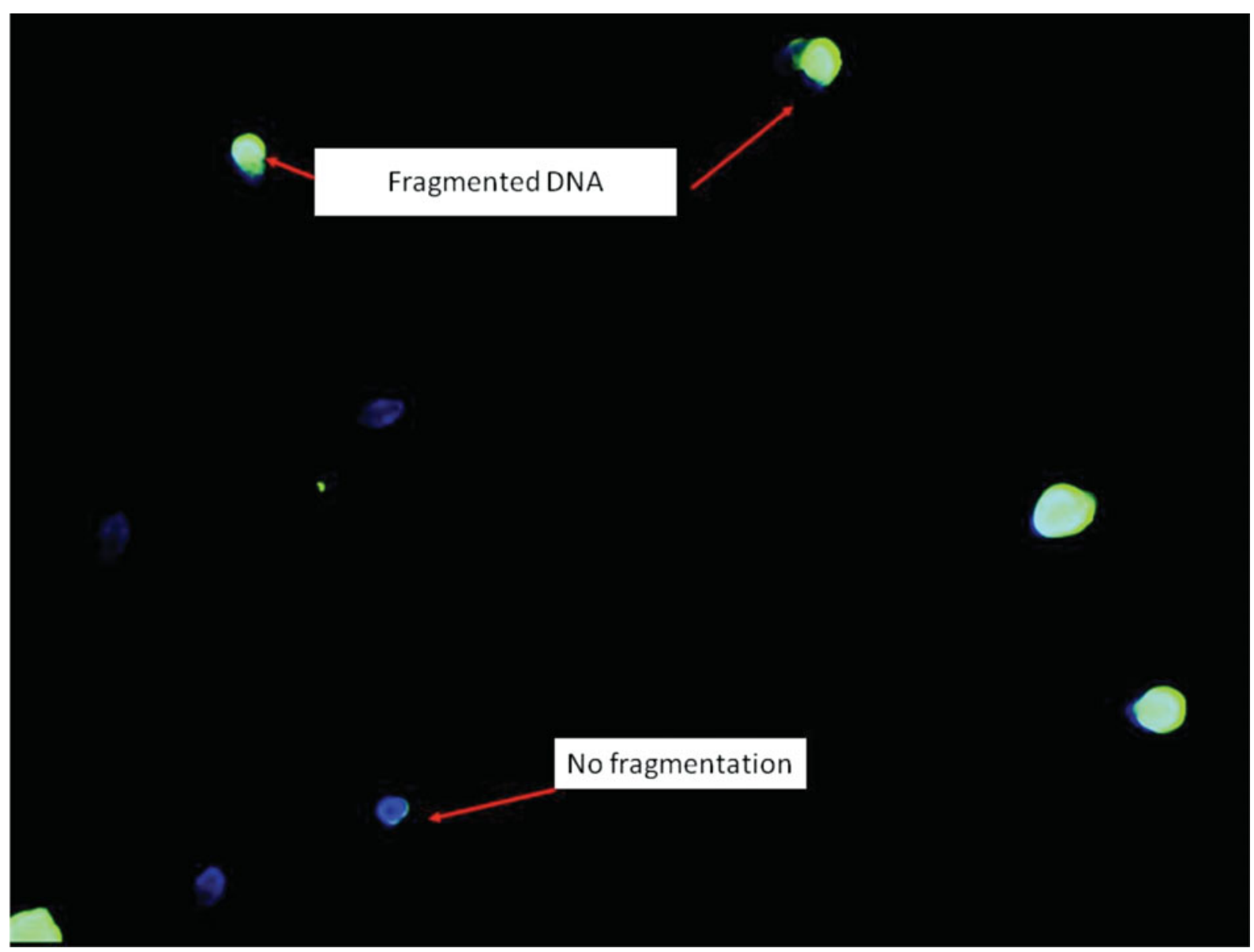

Figure 3 In situ determination of sperm DNA fragmentation with TUNEL. Pale green: fragmented DNA; blue: DNA presenting no fragmentation.

\section{Results}

\section{DNA fragmentation (DFI)}

DNA fragmentation (DFI): means observed TUNEL 18.07, acridine FC: 17.47 .

- Welch two-sample $t$-test; alternative: two-sided T-test: array, confidence interval 95\% [-1.9772; 3.1748]. $P$-value 0.65 : no difference between the two techniques.

- Wilcoxon test: Z-score: -2.57. No difference between the two techniques. Asymptotic significance: 0.1.

Individual variations are shown in Fig 1, in situ image for the test is presented in Fig. 3.

\section{Chromatin decondensation (SDI)}

Chromatin decondensation (SDI): means observed AB: 9.94, acridine orange: 9.01 .

- Welch two-sample $t$-test: confidence interval $95 \%$ $[-0.63 ; 2.47]$. $P$-value 0.24 : no difference between the two tests.
- Wilcoxon test: Z-score: -2.64. Asymptotic significance 0.01 . The tests cannot be considered as similar. Acridine orange gives a lower value for estimation of chromatin decondensation.

Individual variations obtained between the two techniques are shown in Fig. 2, in situ image for the test is presented in Fig. 4.

\section{Discussion}

In situ analysis has two main disadvantages/two obstacles: the number of cells analysed and the operator dependence. It has also to be said that sperm samples can be frozen before FC acridine orange testing for SDI; this is not the case for $\mathrm{AB}$. The samples cannot be frozen before $A B$ testing otherwise the sperm cells are fully (blue) coloured. Automated FC avoids these difficulties, comparing in situ TUNEL versus $\mathrm{FC}$ acridine orange (red fluorescence) on the one hand and in situ $\mathrm{AB}$ versus $\mathrm{FC}$ acridine orange (green fluorescence) on the other hand gives roughly 


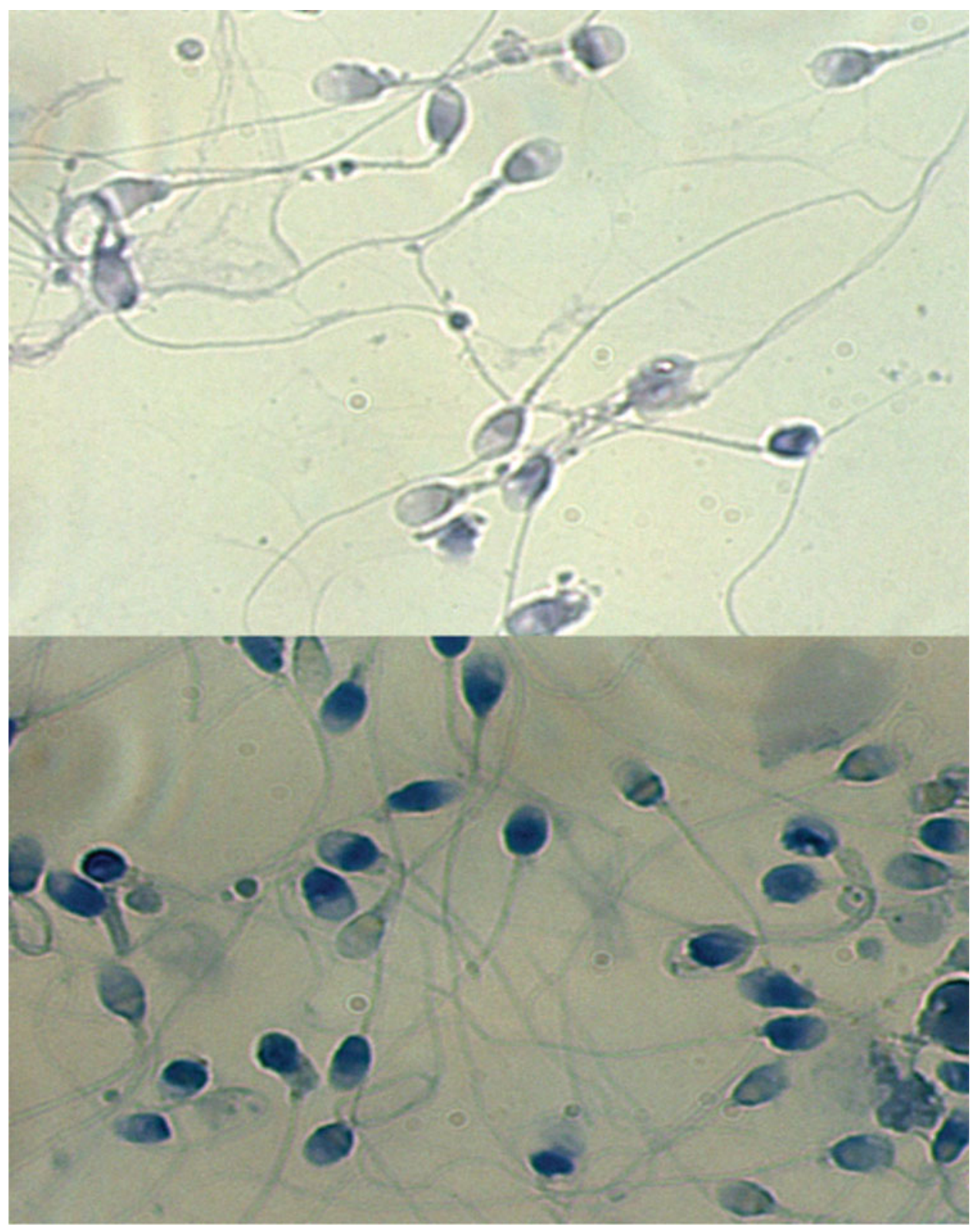

Figure 4 In situ determination of sperm decondensation index (SDI) using aniline blue. Top panel: light staining (SDI: 3\%); bottom panel: heavy staining (SDI: 51\%).

the same results. This is a little bit less valid for AB: the Wilcoxon test give a significant lower quantification with FC acridine orange green fluorescence (SDI), whilst the Welch test leads to a similar quantification.

For sperm DNA fragmentation the distribution in our general population, is, on a yearly basis, the following: using TUNEL DFI $<25 \%$ : $86 \%$; DFI $>25 \%$ : $14 \%$ (1336 patients). But $53 \%$ of the patients have a DFI between $20-25 \%$. An important percentage of the patients consulting for infertility is in this grey zone $20-25 \%$. The pathological values limits are still controversial and may vary according to the techniques employed (Kazerooni et al., 2009,
Sharma et al., 2010, Simon et al., 2011, Lewis et al. 2013). The threshold of $20-25 \%$ for sperm DNA fragmentation for decreased fertility is not far from the consensus: this could be considered as a grey zone where fertility problems start. The upper limit for having a healthy delivery can be considered at $35-40 \%$. The success of ART also depends of the quality of the oocytes and especially the importance of the DNA repair possibilities, i.e. the mRNAs coding for this process (Menezo et al., 2007). This is especially true for fragmentation: the repair mechanisms are complete and iterating in the healthy (young) oocyte. This is less valid for chromatin condensation, 
i.e. tertiary structure: the oocyte has a poor capacity to manage with an abnormal chromatin structure (Menezo et al., 2007). In our overall population, 17\% of patients have a SDI strictly over $25 \%(\mathrm{AB}, 528$ patients). The determination of the 'risky' threshold is not just a matter of semantics. SDI is a neglected parameter, even if it is now clear that chromatin structure is an important parameter for the capacity to produce live births. The negative impact of a high decondensation may occur immediately at the time of fertilization, with the 'no fertilization' syndrome in intra-cytoplasmic sperm injection (ICSI) (Junca et al., 2012) and early developmental arrests (Dattilo et al., 2014), up to miscarriages (Kazerooni et al., 2009). Considering DFI, numerous observations have focused on the risk for the outcome and the next generation (Hemminki et al. 1999, Belloc et al., 2008, Fernández-Gonzalez et al., 2008). This includes an increased risk of cancer for the next generation. In a recent analysis of babies born after ICSI, Davies et al. (2012) demonstrated an increased risk of birth defects. The DFI increases with age and it is obvious that the patients seeking ART are older compared with the general population. Only one-third of our patients consulting for hypofertility seems totally 'clean' of an impairing DNA fragmentation. Moreover, in a recent paper we were able to demonstrate that both DFI and SDI are stable with time in the absence of any treatment (Menezo et al., 2014). In conclusion, FC acridine orange allows us to check simultaneously both sperm DNA fragmentation and decondensation. The determination of these parameters seems increasingly necessary: it has to be clearly said that ICSI does not cure all male fertility problems and may even be hazardous for the next generation, as oxidative stress and imprinting are also linked (Menezo et al., 2013). Finally, it has to be pointed out that the repair capacity of the human oocyte cannot be improved and may be insufficient to overcome paternally borne damage. Therefore, a scientific approach for an in vivo improvement of spermatozoa, via the improvement of the one carbon cycle (Menezo et al., 2014) before ART remains of paramount importance.

\section{References}

Auger, J., Mesbah, M., Huber, C. \& Dadoune, J.P. (1990). Aniline blue staining as a marker of sperm chromatin defects associated with different semen characteristics discriminates between proven fertile and suspected infertile men. Int. J. Androl. 13, 452-62.

Badouard, C., Ménézo, Y., Panteix, G., Ravanat, J.L., Douki, T., Cadet, J. \& Favier, A. (2008). Determination of new types of DNA lesions in human sperm. Zygote 16, 9-13.

Belloc, S., Cohen-Bacrie, P., Benkhalifa, M., Cohen-Bacrie, M., De Mouzon, J., Hazout, A. \& Ménézo, Y. (2008).
Effect of maternal and paternal age on pregnancy and miscarriage rates after intrauterine insemination. Reprod. Biomed. Online 17, 392-7.

Belloc, S., Benkhalifa, M., Junca, A.M., Dumont, M., Bacrie, P.C. \& Ménézo, Y. (2009). Paternal age and sperm DNA decay: discrepancy between chromomycin and aniline blue staining. Reprod. Biomed. Online 19, 264-9.

Cohen-Bacrie, P., Belloc, S., Ménézo, Y.J., Clement, P., Hamidi, J. \& Benkhalifa, M. (2009). Correlation between DNA damage and sperm parameters: a prospective study of 1,633 patients. Fertil. Steril. 9, 1801-5.

Dattilo, M., Cornet, D., Amar, E., Cohen, M. \& Menezo, Y. (2014). The importance of the one carbon cycle in human male fertility: a preliminary clinical report. Reprod. Biol. Endocrinol. (in press).

Davies, M.J., Moore, V.M., Willson, K.J., Van Essen, P., Priest, K., Scott, H., Haan, E.A. \& Chan, A. (2012). Reproductive technologies and the risk of birth defects. New Engl. J. Med. 366, 1803-13.

Eid, L.N., Lorton, S.P. \& Parrish, J.J. (1994). Paternal influence on S-phase in the first cell cycle of the bovine embryo. Biol. Reprod. 51, 1232-7.

Evenson, D.P., Darzynkiewicz, Z. \& Melamed, M.R (1980). Relation of mammalian sperm chromatin heterogeneity to fertility. Science 210, 1131-3.

Fernández-Gonzalez, R., Moreira, P.N., Pérez-Crespo, M., Sánchez-Martín, M., Ramirez, M.A., Pericuesta, E., Bilbao, A., Bermejo-Alvarez, P., de Dios Hourcade, J., de Fonseca, F.R. \& Gutiérrez-Adán, A. (2008). Long-term effects of mouse intracytoplasmic sperm injection with DNAfragmented sperm on health and behavior of adult offspring. Biol. Reprod. 78, 761-72.

Gaspari, L., Chang, S.S., Santella, R.M., Garte, S., Pedotti, P. \& Taioli, E. (2003). Polycyclic aromatic hydrocarbon-DNA adducts in human sperm as a marker of DNA damage and infertility. Mutat. Res. 535, 155-60.

Hamadeh, M.E., Al-Hasani, S., Stieber, M., Rosenbaum, P., Küpker, D., Diedrich, K. \& Schmidt, W. (1996). The effect of chromatin condensation (aniline blue staining) and morphology (strict criteria) of human spermatozoa on fertilization, cleavage and pregnancy rates in an intracytoplasmic sperm injection programme. Hum. Reprod. 11, 2468-71.

Hammoud, S.S., Nix, D.A., Zhang, H., Purwar, J., Carrell, D.T. \& Cairns, B.R. (2009). Distinctive chromatin in human sperm packages genes for embryo development. Nature 460, 473-8.

Hemminki, K., Kyyrönen, P. \& Vaittinen, P. (1999). Parental age as a risk factor of childhood leukemia and brain cancer in offspring. Epidemiology 10, 271-5.

Ji, G., Yan, L., Wu, S., Liu, J., Wang, L., Zhang, S., Shi, L. \& Gu, A. (2013). Bulky DNA adducts in human sperm associated with semen parameters and sperm DNA fragmentation in infertile men: a cross-sectional study. Environ. Health 12, 82-9.

Junca, A., Gonzalez Marti, B., Tosti, E., Cohen, M., De la Fontaine, D., Benkhalifa, M. \& Ménézo, Y. (2012). Sperm nucleus decondensation, hyaluronic acid (HA) binding and oocyte activation capacity: different markers of sperm immaturity? Case reports. J. Assist. Reprod. Genet. 29, 3535. 
Kazerooni, T., Asadi, N., Jadid, L., Kazerooni,, M., Ghanadi, A., Ghaffarpasand, F., Kazerooni, Y. \& Zolghadr, J. (2009). Evaluation of sperm's chromatin quality with acridine orange test, chromomycin A3 and aniline blue staining in couples with unexplained recurrent abortion. J. Assist. Reprod. Genet. 26, 591-6.

Kim, H.S., Kang, M.J., Kim, S.A., Oh, S.K., Kim, H., Ku, S.Y., Kim, S.H., Moon, S.Y. \& Choi, Y.M. (2013). The utility of sperm DNA damage assay using toluidine blue and aniline blue staining in routine semen analysis. Clin. Exp. Reprod. Med. 40, 232-8.

Lewis, S.E., Aitken, J.R., Conner, S.J., Iuliis, G.D., Evenson, D.P., Henkel, R., Giwercman, A. \& Gharagozloo, P (2013). The impact of sperm DNA damage in assisted conception and beyond: recent advances in diagnosis and treatment. Reprod. Biomed. Online 27, 325-37.

Menezo, Y. Jr, Russo, G., Tosti, E., El Mouatassim, S. \& Benkhalifa, M. (2007). Expression profile of genes coding for DNA repair in human oocytes using pangenomic microarrays, with a special focus on ROS linked decays. J. Assist. Reprod. Genet. 24, 513-20.

Menezo, Y., Dale, B. \& Cohen, M. (2010). DNA damage and repair in human oocytes and embryos: a review. Zygote 18, 357-65.

Menezo, Y., Lichtblau, I. \& Elder, K. (2013). New insights into human pre-implantation metabolism in vivo and in vitro. J. Assist. Reprod. Genet. 30, 293-303.

Menezo, Y., Evenson, D., Cohen, M. \& Dale, B. (2014). Effect of antioxidants on sperm genetic damage. Adv. Exp. Med. Biol. 791, 173-89.

Rousseaux, S., Reynoir, N., Escoffier, E., Thevenon, J., Caron, C. \& Khochbin, S. (2008). Epigenetic reprogramming of the male genome during gametogenesis and in the zygote. Reprod. Biomed. Online 16, 492-503.

Sakkas, D., Urner, F., Bianchi, P.G., Bizzaro, D., Wagner, I., Jaquenoud, N., Manicardi, G. \& Campana, A. (1996). Sperm chromatin anomalies can influence decondensation after intracytoplasmic sperm injection. Hum. Reprod. 11, 837-43.

Sellami, A., Chakroun, N., Ben Zarrouk, S., Sellami, H., Kebaili, S., Rebai, T. \& Keskes, L (2013). Assessment of chromatin maturity in human spermatozoa: useful aniline blue assay for routine diagnosis of male infertility. Adv. Urol. 2013, 578631. doi: 10.1155/2013/578631. Epub ahead of print.

Sharma, R.K., Sabanegh, E., Mahfouz, R., Gupta, S., Thiyagarajan, A. \& Agarwal, A. (2010). TUNEL as a test for sperm DNA damage in the evaluation of male infertility. Urology 76, 1380-6.

Simon, L., Lutton, D., McManus, J. \& Lewis, S.E. (2011). Sperm DNA damage measured by the alkaline Comet assay as an independent predictor of male infertility and in vitro fertilization success. Fertil. Steril. 95, 652-7.

Ward, W.S. (2010). Function of sperm chromatin structural elements in fertilization and development. Mol. Hum. Reprod. 16, 30-6.

Ward, F., Rizos, D., Corridan, D., Quinn, K., Boland, M. \& Lonergan, P. (2001). Paternal influence on the time of first embryonic cleavage post insemination and the implications for subsequent bovine embryo development in vitro and fertility in vivo. Mol. Reprod. Dev. 60, 47-55.

World Health Organization (2004). DHS Comparative Reports No. 9, Infecundity, Infertility, and Childlessness in Developing Countries. Www.who.int/ reproductivehealth/topics/infertility/DHS-CR9. 